compared with usual care (nonsteroidal antiinflammatory drugs, physiotherapy, or both) in AS.

All patients had a Bath Ankylosing Spondylitis Disease Activity Index score of $\geq 4$. Probabilities of toxicity of TNF inhibitors, efficacy and relapse were derived from two European randomized trials. Benefits and costs used in the analysis were derived from a 2-year observational Dutch cohort.

The quality-adjusted life years (QALYs) gained by treatment were 2.89, 3.16 and 3.11 for usual care, etanercept and infliximab, respectively. The estimated incremental costs of TNF inhibitors per QALY compared with standard care were $€ 42,914-123,761$ for etanercept, and $€ 67,207-237,010$ for infliximab.

Data showed that the cost per QALY of etanercept and infliximab are greater than the implicit thresholds of cost per QALY that are applied in the US, the UK and the Netherlands; however, this study was hampered by limited data on the natural progression of AS and the long-term effects of TNF inhibitors. Future investigations to improve cost-benefit analyses, and to identify subgroups of patients for whom anti-TNF treatment is cost-effective, are warranted.

Rachel Murphy

Original article Boonen A et al. (2006) Markov model into the cost-utility over five years of etanercept and infliximab compared with usual care in patients with active ankylosing spondylitis. Ann Rheum Dis 65: 201-208

\section{Prednisone use is linked to pneumonia risk in patients with rheumatoid arthritis}

Infectious pneumonia is a major cause of death in patients with rheumatoid arthritis (RA), but this relationship has not been specifically studied. Wolfe et al. aimed to find the incidence of pneumonia among these patients, and to determine if treatment with prednisone, disease-modifying antirheumatic drugs, or anti-tumor necrosis factor (anti-TNF) agents increases the risk of pneumonia. The team assessed 16,788 patients with RA from the National Data Bank for Rheumatic Diseases, every 6 months for 3.5 years.

The incidence of pneumonia was 17 cases per 1,000 patient-years (95\% Cl 16.4-19.1) for the whole cohort, and 14.7 cases per 1,000 patient-years $(95 \% \mathrm{Cl} 13.1-16.4)$ for the subgroup not enrolled in drug-safety registriesa population more representative of patients in the community than the cohort is as a whole.

Several nontreatment variables were associated with increased pneumonia risk, including male sex, older age, comorbidities-such as diabetes and smoking history-and number of therapeutic agents taken for RA. After adjustment for covariates, the only treatments associated with a significant risk of pneumonia were prednisone (hazard ratio 1.7, 95\% Cl 1.5-2.1) and leflunomide (hazard ratio $1.3,95 \% \mathrm{Cl} 1.0$ 1.5). The association with prednisone was doserelated and remained robust when all treatment variables were analyzed simultaneously.

Prednisone is commonly used to treat RA, so there are important implications if it increases the risk of pneumonia. Interestingly, although TNF is thought to be involved in infection response, anti-TNF therapies did not increase pneumonia risk.

Chrissie Giles

Original article Wolfe F et al. (2006) Treatment for rheumatoid arthiritis and the risk of hospitalization for pneumonia: associations with prednisone, disease-modifying antirheumatic drugs, and anti-tumor necrosis factor therapy. Arthritis Rheum 54: 628-634

\section{Mirthful laughter affects cytokine levels in patients with rheumatoid arthritis}

Cytokines have a primary role in mediating the underlying pathologic processes of inflammation and tissue destruction in rheumatoid arthritis (RA), and increasing evidence suggests that interaction between the effects of stress and the immune system is crucial in the etiology and progression of autoimmune diseases. In a Japanese study, Matsuzaki et al. investigated the effects of mirthful laughter on cytokine levels in patients with RA.

Mirthful laughter was induced by a $1 \mathrm{~h}$ comic story listened to by 41 RA patients and 23 healthy participants. Blood samples were collected within the $10 \mathrm{~min}$ preceding and following the story, and levels of (proinflammatory) interleukin (IL)-6, tumor necrosis factor, and (anti-inflammatory) IL-4 and IL-1 receptor antagonists were measured by enzyme-linked immunosorbent assay. The patients with RA were divided into two groups according to their C-reactive-protein level (as a surrogate 